\title{
Comparative transcriptome analysis and marker development of two closely related Primrose species (Primula poissonii and Primula wilsonii)
}

\author{
Lu Zhang ${ }^{1,2}$, Hai-Fei Yan ${ }^{1}$, Wei Wu ${ }^{1 *}$, Hui Yu ${ }^{1}$ and Xue-Jun Ge ${ }^{1}$
}

\begin{abstract}
Background: Primula species are important early spring garden plants with a centre of diversity and speciation in the East Himalaya-Hengduan Mountains in Western China. Studies on population genetics, speciation and phylogeny of Primula have been impeded by a lack of genomic resources. In the present study, we sequenced the transcriptomes of two closely related primrose species, Primula poissonii and Primula wilsonii, using short reads on the Illumina Genome Analyzer platform.

Results: We obtained 55,284 and 55,011 contigs with N50 values of 938 and 1,085 for P. poissonii and P. wilsonii, respectively, and 6,654 pairs of putative orthologs were identified between the two species. Estimations of non-synonymous/synonymous substitution rate ratios for these orthologs indicated that 877 of the pairs may be under positive selection (Ka/Ks > 0.5), and functional enrichment analysis revealed that significant proportions of the orthologs were in the categories DNA repair, stress resistance, which may provide some hints as to how the two closely related Primula species adapted differentially to extreme environments, such as habitats characterized by aridity, high altitude and high levels of ionizing radiation. It was possible for the first time to estimate the divergence time between the radiated species pair, $P$. poissonii and $P$. wilsonii; this was found to be approximately $0.90 \pm 0.57 \mathrm{Mya}$, which falls between the Donau and Gunz glaciation in the Middle Pleistocene. Primers based on 54 pairs of orthologous SSR-containing sequences between the two Primula species were designed and verified. About half of these pairs successfully amplified for both species. Of the 959 single copy nuclear genes shared by four model plants (known as APVO genes), 111 single copy nuclear genes were verified as being present in both Primula species and exon-anchored and intron-spanned primers were designed for use.

Conclusion: We characterized the transcriptomes for the two Primula species, and produced an unprecedented amount of genomic resources for these important garden plants. Evolutionary analysis of these two Primula species not only revealed a more precise divergence time, but also provided some novel insights into how differential adaptations occurred in extreme habitats. Furthermore, we developed two sets of genetic markers, single copy nuclear genes and nuclear microsatellites (EST-SSR). Both these sets of markers will facilitate studies on the genetic improvement, population genetics and phylogenetics of this rapidly adapting taxon.
\end{abstract}

Keywords: Adaptive radiation, East Himalaya-Hengduan Mountains, EST-SSR, Single copy nuclear gene

\footnotetext{
*Correspondence: wuei@scib.ac.cn

'Key Laboratory of Plant Resource Conservation and Sustainable Utilization, South China Botanical Garden, Chinese Academy of Science, Guangzhou 510650, China

Full list of author information is available at the end of the article
} 


\section{Background}

Adaptive radiation, 'the rise of a diversity of ecological roles and attendant adaptations in different species within a lineage' is one of the most important processes bridging the gap between ecology and evolution [1]. Usually, the genetic divergence between species within adaptive radiations is very small, and only a handful of genes with large effects are responsible for differences in ecologically significant traits and reproductive isolation between species. Due to the lack of availability of molecular markers for rapidly evolving taxa, especially from nuclear genome, most plant molecular systematic studies on adaptive radiation have hitherto failed to provide resolved phylogenies. The same is true for speciation studies, which rely heavily on there being sufficient intraspecific genetic variation. Moreover, we still have little understanding of how divergent natural selection may have acted on the genomes of such species within the short evolutionary time span since their common ancestor [2].

Transcriptome analysis is not only an effective way to study gene expression in specific tissues at specific time, and it also provides unprecedented opportunities to address comparative genomic-level questions for nonmodel organisms. RNA-sequencing (RNA-seq) is an efficient new technology for large scale transcriptome investigations. With the rapid development of nextgeneration sequencing (NGS), RNA-sequencing becomes more efficient and less expensive, and is increasingly being used to study the evolutionary origins and ecology of nonmodel plants [3,4]. For instance, a large number of microsatellite markers or single-copy nuclear genes in yam (Dioscorea alata) [5], buckwheat (Fagopyrum) [6] and big sagebrush (Artemisia tridentata) [7] have been identified by making use of RNA-sequencing. Since RNA-sequencing is still somewhat expensive at present, few RNA-seq studies to date have included for more than one species at the same time [6,7]. However, comparative RNA-sequencing studies between closely related species can in principle not only provide additional genomic resources such as genusspecific SSR primers or single copy nuclear gene primers, but also give information about the processes of speciation or adaptive evolution, e.g. divergence time estimations, or detection of adaptive loci.

Primula with around 430 species, is one of the three great garden genera [8], and southwestern China, in which ca. 187 species of the genus are distributed, is its diversity centre $[9,10]$. In this region Primula shows a typical patterns of adaptive evolution and explosive speciation; however, research has been hampered by the fact that few Primula genomic resources are available. Up to now, only a few SSR primers from the three Primula species $P$. vulgaris, $P$. obconica, and P. sieboldii have been developed [11-13], and only one large EST collection, consisting of 5,651 ESTs generated from Primula sieboldii were available [13]. Paucity of genetic data such as genome sequences, transcriptome sequences and associated molecular markers has made Primula breeding or evolutionary analysis a challenging task.

Primula section Proliferae Pax, which contains ca. 25-30 species and is centred on southwestern China, is regarded as a taxonomically well-known group circumscribed by possession of numerous whorls of flowers [14]. Within this section, Primula wilsonii and P. poissonii are two closely related species with very similar morphological characters, and the two diagnostic characters used to distinguish them are the corolla structure and the aromatic fragrance of fresh leaves; for $P$. wilsonii, the fresh leaves are fragrant and corolla limbs are slightly opened, whereas, $P$. poissonii has no obvious fragrance and widely opened corolla limbs [14]. These closely related species represent a useful resource for addressing two questions: how did Primula species in southwestern China radiate within a short period of time, and what was the driving force underlying the process of rapid adaptive evolution? As the first step towards answering these questions, in this study, we obtained transcriptomes for Primula poissonii and P. wilsonii using the Illumina platform, and carried out a comprehensive analysis of them. Our aims were to 1) characterize the transcriptomes of $P$. poissonii and $P$. wilsonii, and increase the genetic resources available for Primula breeding or evolutionary analysis; 2) determine the evolutionary dynamics of the two species, including obtaining a divergence time estimation, signatures of adaptive evolution between the two species; and 3) discover genus-specific SSR markers and single-copy nuclear gene markers from both species.

\section{Results and discussion}

De novo assembly and functional annotation of contigs

After cleaning of raw sequences, ca. 55 million 75-bp paired-end reads were obtained for both $P$. poissonii and $P$. wilsonii,. We obtained 55,284 contigs with a mean length of 655 and an N50 value of 938 for P. poissonii, and 55,011 contigs with a mean length of 722 and an N50 value of 1,085 for $P$. wilsonii (Table 1). Contig with lengths between 200 and 500 bp were overrepresented, making up about $56 \%$ of the total number of contigs for P. poissonii, and $53 \%$ for P. wilsonii, the next most abundant size class was 500-1000 bp, constituting about $24 \%$ and $24 \%$ of the total, respectively (Figure 1). To evaluate the quality of de novo assembly, we obtained a total of 16,346 peptide sequences from Vitis. For P. poissonii, 34,660 contigs were annotated to 13,800 (84.4\%) Vitis proteins, of which, $6,869(49.8 \%)$ proteins were covered for at least $70 \%$ of the full length. For $P$. wilsonii, 34,930 contigs were annotated to 13,955 (85.4\%) Vitis proteins, of which, $7693(55.1 \%)$ proteins were covered at least 
Table 1 Summary of assembly and annotation results for $P$. poissonii and $P$. wilsonii using Trinity

\begin{tabular}{lcc}
\hline & P. poissonii & P. wilsonii \\
\hline Total number of reads & $55,056,996 \times 2$ & $55,468,564 \times 2$ \\
Total number of contigs & 55,284 & 55,011 \\
Mean length of contigs & 655 & 722 \\
Median length of contigs & 432 & 469 \\
N50 value of contigs & 938 & 1085 \\
Length range of contigs & $200 \sim 16,932$ & $200 \sim 12,384$ \\
GC content & $41.3 \%$ & $41.2 \%$ \\
Contigs with BLASTX hit & $36,239(65.6 \%)$ & $35,857(65.1 \%)$ \\
Contigs with annotation & $28,435(51.4 \%)$ & $28,302(51.4 \%)$ \\
\hline
\end{tabular}

$70 \%$ of the full length. The GC content for P. poissonii and $P$. wilsonii sequences is $41.3 \%$ and $41.2 \%$, respectively.

In BLASTX homology research with the cutoff E-value set at 1E-6, 36,239 contigs (65.6\%) for P. poissonii and 35,857 contigs $(65.1 \%)$ for P. wilsonii gave hits. For both species, the three top-hit species were Vitis vinfera, Populus trichocarpa and Ricinus communis (Figure 2). A total of 28,435 (51.4\%) and 28,302 (51.4\%) contigs were assigned at least one GO terms for $P$. poissonii and $P$. wilsonii, respectively. For the biological process category, the two mostly highly represented terms among the 23 level-2 categories were cellular process and metabolic process; for the molecular function category, among the 13 level-2 categories, binding and catalytic activity were overrepresented; for the 14 level- 2 categories in the cellular component category, cell, cell part and organelle were the most abundant terms (Figure 3). These categories were similarly distributed in both species.

\section{Orthologous contigs, substitution rates, and} transcriptome divergence between two Primula species We identified 28,482 pairs of putative orthologous contigs between $P$. poissonii and $P$. wilsonii using the reciprocal best hit method with BLASTN algorithm. After incorporating the Vitis peptide sequences [15], 7,006 pairs of putative orthologs were obtained using the RBM triangulation method [16]. This reduction in ortholog numbers was caused mainly by the exclusion of the relatively young orthologs specific to Primula, which were discarded as being low similarity to Vitis. After excluding alignments with unexpected stop codons, lengths less than $150 \mathrm{bp}$ or Ks values above 0.1 , 6,654 pairs of orthologs were retained for subsequent analysis.

Using the Vitis proteins as reference, the coding regions of 6,654 pairs of orthologs from P. poissonii and P. wilsonii

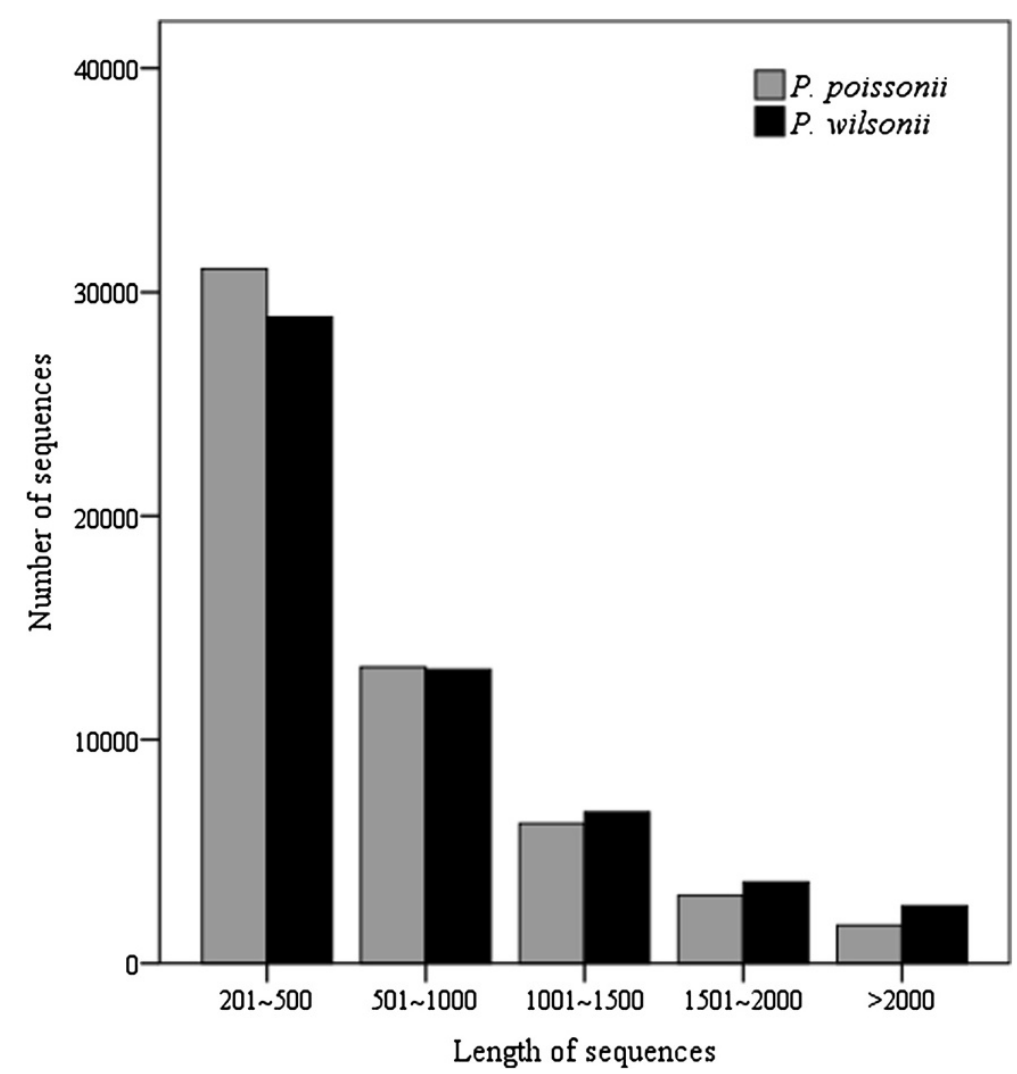

Figure 1 Length distributions of contigs for two Primula species. Grey bar, P. poissonii; black bar, P. wilsonii. 


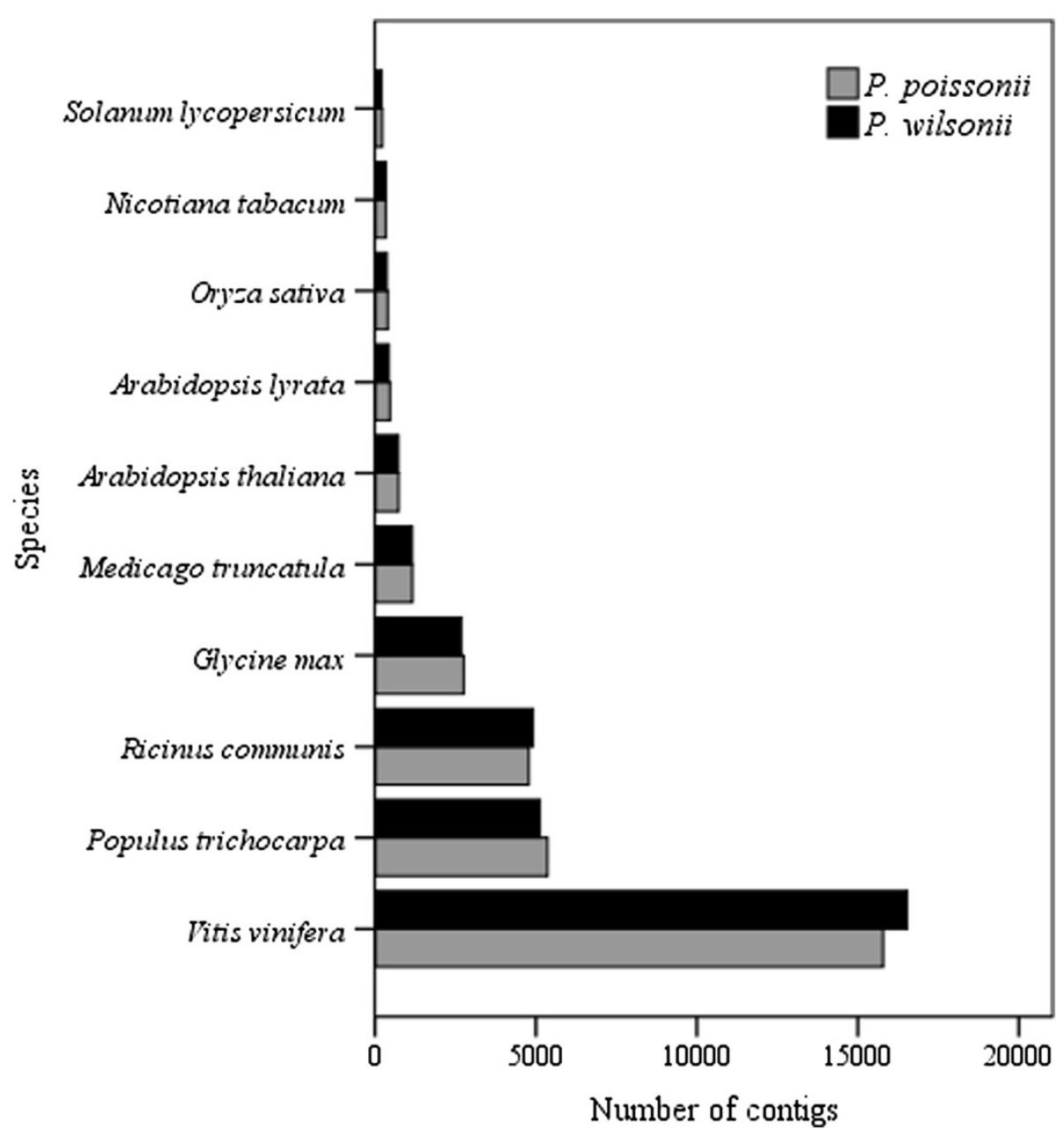

Figure 2 Top-hit species distribution for sequences from two Primula species submitted BLASTX against the NCBI-nr. database. Grey bar, P. poissonii; black bar, P. wilsonii.

were extracted, in some cases, $5^{\prime}$-UTR (1,315 pairs of orthologs) or 3 '-UTR (2,051 pairs of orthologs) were also determined. The average genetic divergence of coding regions between the two Primula species is $0.011 \pm 0.007$ according to the K2P model. The genetic divergence between the two species is $0.019 \pm 0.017$ for $5^{\prime}$-UTR and $0.018 \pm 0.013$ for 3 '-UTR regions. The accelerated substitution rate observed in the 5'UTR and 3' UTR relative to the coding region, is indicative of relaxed functional constraint on the evolution of the UTR than on the coding region at the genome level, which is consistent with the evidence from other model species-pairs [17].

Among the 6,654 pairs of orthologs between $P$. poissonii and $P$. wilsonii, 165 pairs were identical, 1,327 pairs had only either synonymous or nonsynonymous substitutions, and 5,162 pairs had both types of substitutions, for which the $\mathrm{Ka} / \mathrm{Ks}$ ratio were calculated. The mean values of $\mathrm{Ka}$,
$\mathrm{Ks}$, and the $\mathrm{Ka} / \mathrm{Ks}$ ratio of all orthologous pairs were $0.007 \pm 0.005,0.027 \pm 0.017$ and $0.322 \pm 0.324$, respectively. Of the 5,162 pairs of orthologs, 233 pairs with a Ka/Ks value $>1$ were found. Taking a more appropriate threshold of 0.5 for the $\mathrm{Ka} / \mathrm{Ks}$ ratio as an indicator of positive selection [18], 644 pairs with a $\mathrm{Ka} / \mathrm{Ks}$ value between 0.5 and 1 were also found.

Peaks in the Ks value distribution of orthologs between closely related species often indicates speciation events [19], and this approach has been successfully used in the inference of such events [20]. In this study, a peak of Ks distribution between $P$. poissonii and $P$. wilsonii was observed at $0.027 \pm 0.017$ (Figure 4). The low level of Ks between $P$. poissonii and $P$. wilsonii indicated their close relationship and confirmed the previous taxonomic treatment. Based on the data derived from ESTs of Asteraceae and several model plants provided by Kane 

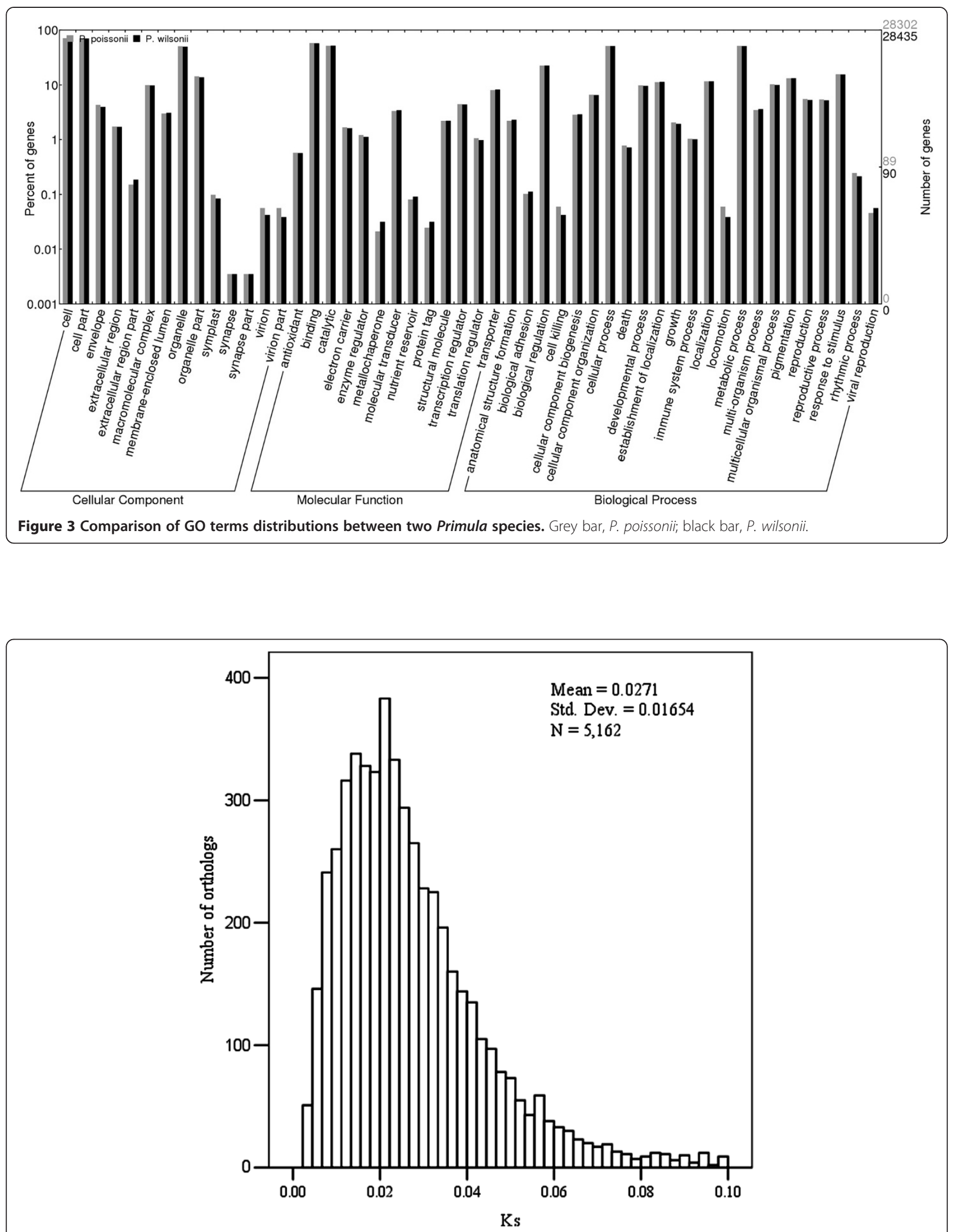

Figure 4 The Ks distribution of orthologs between P. poissonii and $P$. wilsonii, their divergence is shown by the peak of Ks at $0.03 \pm 0.017$. 
[21] (personal communication), we found a mean Ks value of $0.03 \sim 0.10$ between congeneric species. According to these criteria, the differentiation between $P$. poissonii and $P$. wilsonii is obviously very recent.

The peak synonymous rates (Ks) for orthologous transcript pairs can be used to estimate the times of divergence between species. To obtain a rough estimate of the divergence time ( $\mathrm{T}$ ) between $P$. poissonii and $P$. wilsonii, we followed the simple formula: $T=K / 2 r$ [22], where $r$ is the mean rate of synonymous substitution, and is considered to be $1.5 \mathrm{E}-8$ substitutions/synonymous site/year for all dicots [23]; $\mathrm{K}$ is genetic divergence expressed in terms of mean number of synonymous substitutions between orthologs. The age of the speciation event between $P$. poissonii and $P$. wilsonii is approximately $0.90 \pm 0.57$ Mya, which falls between the Donau and Gunz glaciation in the Middle Pleistocene. Bearing in mind disputes about the substitution rate [24], this divergence time is only an appropriate estimate based on the coding region of orthologous genes, nonetheless, it is useful because there is as yet no adequate fossil dating the divergence of the two Primula species.
Functions under positive selection and implications for adaptive evolution between two Primula species

In enrichment analyses, we categorized the orthologs into two datasets: a test dataset with $\mathrm{Ka} / \mathrm{Ks}>0.5$, and a reference dataset with $\mathrm{Ka} / \mathrm{Ks}<0.5$. In an analysis of $\mathrm{GO}$ terms with at least five hits, 20 GO-terms annotated to 98 pairs of orthologs were found to be over-represented (Fisher's exact test, $P$-value $<0.05$ ) in the test dataset (Table 2, Additional file 1: Table S1). For the 98 selected genes, we used BLASTX search to find their orthologous genes in Arabidopsis, and the results showed that the genes with function in DNA repair, stress resistance were overrepresented (Tables 2 and 3). Among the candidate genes under positive selection with $\mathrm{Ka} / \mathrm{Ks}>0.5$, almost one-quarter of them were involved in the DNA repair. DNA repair is essential for maintenance of genomic stability in all organisms. For instance, in our study, the ortholog pw11166, and pw24180 were found to be homologous to $K u 70$ and $K u 80$, which are involved in the repair of DNA double-strand breaks (DBSs) by nonhomologous end joining (NHEJ) [25]; other orthologs, pw42431, pw06163, pw54400 were homologous to SMC5,

Table 2 Gene Ontology terms significantly over-represented in the test dataset versus the reference set

\begin{tabular}{|c|c|c|c|c|}
\hline GO ID & GO term & P-value (Fisher's exact test) & Frequency in test set & Frequency in reference set \\
\hline & Biological Process & & & \\
\hline GO:0007059 & chromosome segregation & 0.05 & 3 & 3 \\
\hline GO:0016790 & thiolester hydrolase activity & 0.03 & 6 & 11 \\
\hline GO:0035966 & response to topologically incorrect protein & 0.03 & 3 & 2 \\
\hline GO:0006281 & DNA repair & 0.04 & 14 & 43 \\
\hline GO:0006289 & nucleotide-excision repair & 0.01 & 4 & 2 \\
\hline GO:0032012 & regulation of ARF protein signal transduction & 0.05 & 3 & 3 \\
\hline GO:0006544 & glycine metabolic process & 0.05 & 3 & 3 \\
\hline GO:0042542 & response to hydrogen peroxide & 0.04 & 5 & 8 \\
\hline GO:0022613 & ribonucleoprotein complex biogenesis & 0.03 & 11 & 28 \\
\hline GO:0071843 & cellular component biogenesis at cellular level & 0.03 & 14 & 41 \\
\hline GO:0006979 & response to oxidative stress & 0.04 & 15 & 47 \\
\hline GO:0006974 & response to DNA damage stimulus & 0.02 & 16 & 47 \\
\hline \multirow[t]{2}{*}{ GO:0044085 } & cellular component biogenesis & 0.05 & 27 & 102 \\
\hline & Molecular Function & & & \\
\hline GO:0008173 & RNA methyltransferase activity & 0.05 & 5 & 9 \\
\hline GO:0004221 & ubiquitin thiolesterase activity & 0.03 & 4 & 5 \\
\hline GO:0003960 & NADPH: quinone reductase activity & 0.03 & 3 & 2 \\
\hline GO:0016790 & thiolester hydrolase activity & 0.03 & 6 & 11 \\
\hline \multirow[t]{2}{*}{ GO:0035091 } & phosphatidylinositol binding & 0.05 & 5 & 9 \\
\hline & Cellular Component & & & \\
\hline GO:0015934 & large ribosomal subunit & 0.03 & 5 & 9 \\
\hline GO:0005802 & trans-Golgi network & 0.04 & 3 & 2 \\
\hline
\end{tabular}


Table 3 Partial list of candidate orthologs under positive selection between $P$. wilsonii and $P$. poissonii

\begin{tabular}{llllr}
\hline P. wilsonii & P. poissonii & Ka/Ks & Arabidopsis thaliana gene accession & Descriptions \\
\hline pw11166 & pp53622 & 1.902 & AT1G16970 & KU70, atp-dependent dna helicase 2 subunit ku70 \\
pw24180 & pp53932 & 1.201 & AT1G48050 & KU80, atp-dependent dna helicase 2 subunit ku80-like \\
pw42431 & pp10104 & 2.016 & AT5G15920 & SMC5, structural maintenance of chromosomes protein \\
pw06163 & pp48369 & 1.087 & AT2G40550 & ETG1, mini-chromosome maintenance complex-binding \\
pw54400 & pp05772 & 1.131 & AT2G24490 & ROR1, replicon protein a2 \\
pw23141 & pp39494 & 1.073 & AT4G31870 & GPX7, glutathione peroxidase \\
pw12283 & pp40105 & 1.726 & AT1G13180 & ARP3, actin-related protein 3 \\
pw37795 & pp02860 & 2.329 & AT1G11755 & LEW1, nogo-b receptor-like \\
pw10616 & pp13461 & 3.711 & AT1G45976 & SBP1, s-ribonuclease binding protein 1 \\
pw09970 & pp42238 & 1.106 & AT3G11050 & FER2, ferritin subunit precursor \\
pw13953 & pp20861 & 0.777 & AT3G54340 & AP3, mads-domain transcription factor \\
pw42171 & pp17454 & 1.099 & AT2G47460 & MYB12, transcription factor myb12 \\
\hline
\end{tabular}

ETG1, ROR1, respectively, which are all involved in DNA repair by homologous recombination [26-28]. The finding that gene families $K u$, and $S M C$ have been under positive selection gives an indication of why $P$. poissonii adapted to the habitats of higher altitude and ionizing radiation than did P. wilsonii.

Some orthologs related to abiotic stress were also found to be positively selected. For example, pw23141 is homologous to GPX7, which regulates cellular photooxidative tolerance and immune response [29]; pw12283 is homologus to $A R P 3$, related to light-induced stomatal opening [30]; pw37795 is homologous to $L E W 1$, the product of which catalyzes the biosynthesis of dolichol [31] and confers acclimation to drought stress, which may partially explain why the two Primula species were able to inhabit habitats with different level of aridity; pw10616 is homologous to SBP1 and pw09970 is homologous to FER2, which are involved in the cadmium stress [32] and iron deficiency [33], respectively; these results shed further light on how the two Primula species differentially adapted to extreme environments. In addition, two positively selected genes are worth notice, one gene pw13953, is homologous to $A P 3$, a key component in the $\mathrm{ABC}$ mode of flower development [34], and may provide a clue about the origin of the differences in corolla structure between P. poissonii and P. wilsonit; the other gene pw42171, is homologous to $M Y B 12$, which functions as a $R 2 R 3-M Y B$ transcription factor in phenylpropanoid biosynthesis [35], also may give some hints on the leaf fragrance differentiation between $P$. poissonii and $P$. wilsonii.

Overall, in this study, we detected a dozens of gene under positive selection between the Primula species pairs, and these findings will not only shed light on how differentiations between two Primula species occurs, but also open the door to increased understanding of how plants living in plateau environments adapt to different characteristics of high altitude, such as strong radiation, aridity and so on.

\section{Identification of microsatellites and single copy genes}

Usually, SSR markers derived from expressed sequence tags (EST-SSRs) are more transferable between species than random genomic SSRs, and they are more advantageous for revealing adaptive differentiations at the population level. Traditional strategies for SSR marker development are labour-intensive and costly. In the case of Primula, up to now, only a few microsatellite primers have been available for Primula obconica, $P$. sieboldii and $P$. vulgaris [11-13], and this has impeded genetic analysis of this important garden plant. Based on the two Primula transcriptomes, 7,571 and 8,272 SSRs were found in $P$. poissonii and $P$. wilsonii, respectively. The most abundant repeat types were dinucleotides followed by trinucleotides (Table 4). The dominant classes of sequence repeat in the contigs were AG/CT, AT/TA and AC/GT, followed by AAG/ CTT repeats (Figure 5), and most SSRs located close to the ends of contigs and were not suitable for primer design (Figure 6). In order to maximize the universal applicability of markers developed in this study and hence reduce their cost, we searched for SSRs in the 6,654 pairs of putative orthologous contigs, and found 1,207 SSRs distributed among 1,073 pairs of contigs (Table 4). Taking only those with a repeat-unit length of at least $16 \mathrm{bp}, 421$ pairs of SSRs contained in 342 pairs of orthologs were selected for primer design, and 54 pairs of sequences with conserved, sufficiently long flanking sites were used to design primers successfully (Additional file 1: Table S2). To evaluate the reliability of these primers, we tested 36 out of the 54 pairs and 24 pairs produced clear fragments with the expected sizes in both Primula species (Figure 7). 
Table 4 Summary of microsatellite loci in Primula poissonii and $P$. wilsonii

\begin{tabular}{|c|c|c|c|c|c|c|c|}
\hline Dataset & $\begin{array}{c}\text { Contigs containing } \\
\text { SSRs }\end{array}$ & SSRs & $\begin{array}{c}\text { Di-nucleotide } \\
\text { repeats }\end{array}$ & $\begin{array}{l}\text { Tri-nucleotide } \\
\text { repeats }\end{array}$ & $\begin{array}{c}\text { Tetra-nucleotide } \\
\text { repeats }\end{array}$ & $\begin{array}{c}\text { Penta-nucleotide } \\
\text { repeats }\end{array}$ & $\begin{array}{l}\text { Hexa-nucleotide } \\
\text { repeats }\end{array}$ \\
\hline P. poissonii & 6363 & 7571 & 6245 & 1158 & 88 & 51 & 29 \\
\hline P. wilsonii & 6981 & 8272 & 6833 & 1249 & 85 & 65 & 40 \\
\hline ortholog pairs & 1073 & 1207 & 1046 & 157 & 3 & 1 & 0 \\
\hline SSRs (repetitive units $>16$ bp) & 342 & 421 & 288 & 89 & 3 & 1 & 0 \\
\hline
\end{tabular}

One noteworthy fact about the SSR primer development based on the Illumina platform is the lower proportion of contigs suitable for primer design compared with Sanger sequencing, in our study, 367 out of the 421 pairs of contigs with SSRs were excluded from primer design because they had insufficient flanking regions caused by NGS assembly algorithms or sequencing [36]. As an alternative, the 454/ Roche sequencing platform, which delivers longer reads, has promise as a way of reducing bias.

Using the APVO gene sets [37] to carry out TBLASTN queries against our Primula dataset orthologs, 612 of the APVO genes were found to give hits against orthologous contigs between $P$. poissonii and $P$. wilsonii over at least $600 \mathrm{bp}$; these are most likely to be single copy genes in the two Primula species. When we set a threshold identity of $75 \%$ with Arabidopsis thaliana and specified facultative intron sizes not less than $300 \mathrm{bp}$ in Arabidopsis thaliana, we were successful in obtaining primers for 111 of the 612 APVO genes (Additional file 1: Table S3); we randomly selected four primer pairs to test, and of these, three of the pairs amplified successfully and when the products were sequenced directly, they yielded the expected gene model, and only one primer pair failed due to the presence of extremely long intron variants, which made the products unsuitable for Sanger sequencing.. The availability from this study of dozens of single copy nuclear genes with heterogeneous rates of variation, will undoubtedly facilitate phylogeny resolution for the radiative Primula species, and open a doorway to understanding the dynamics of speciation using a population genetics approach.

We developed sets of two types of molecular markers, and these two widely-used marker types, each of which

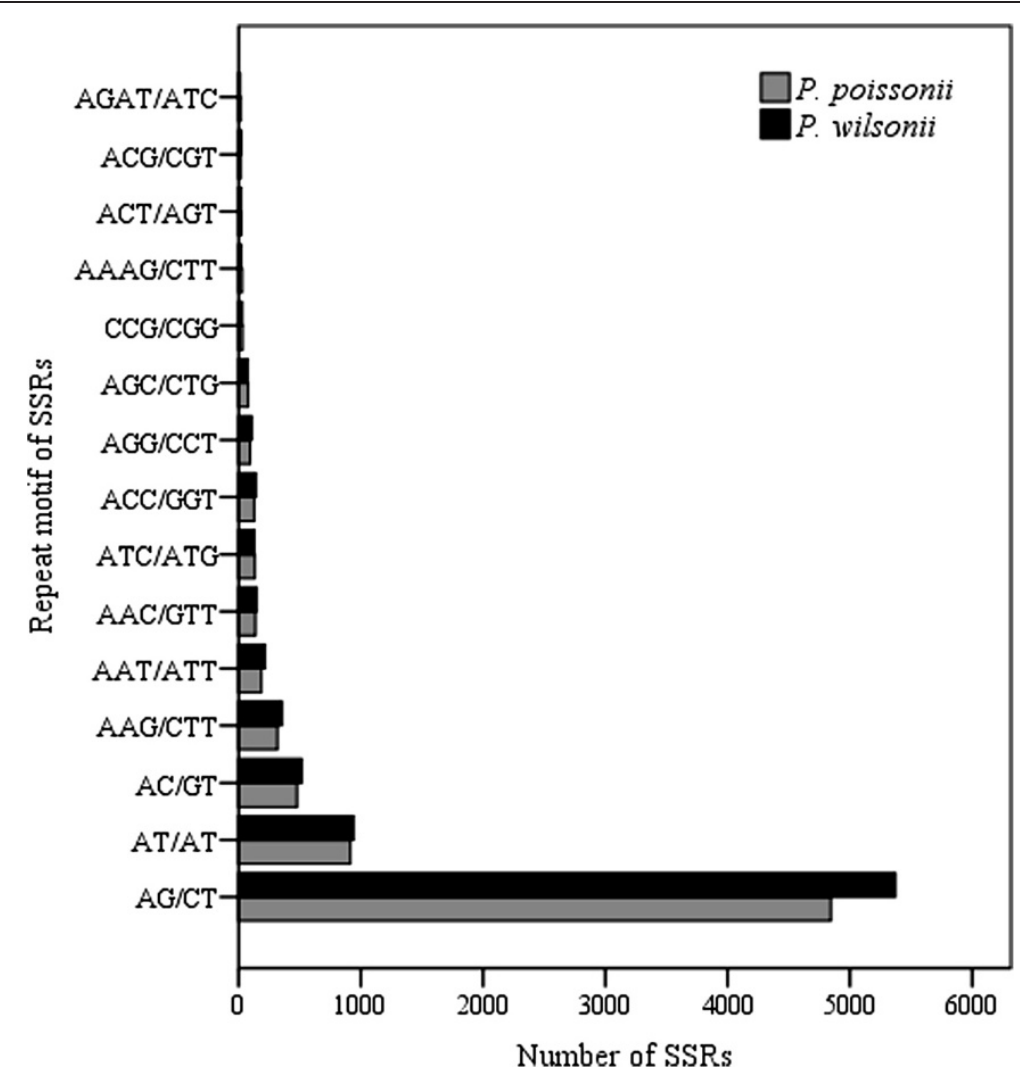

Figure 5 Comparison of the distribution of SSR repeat motifs between two Primula species. Grey bar, P. poissonii; black bar, P. wilsonii. 


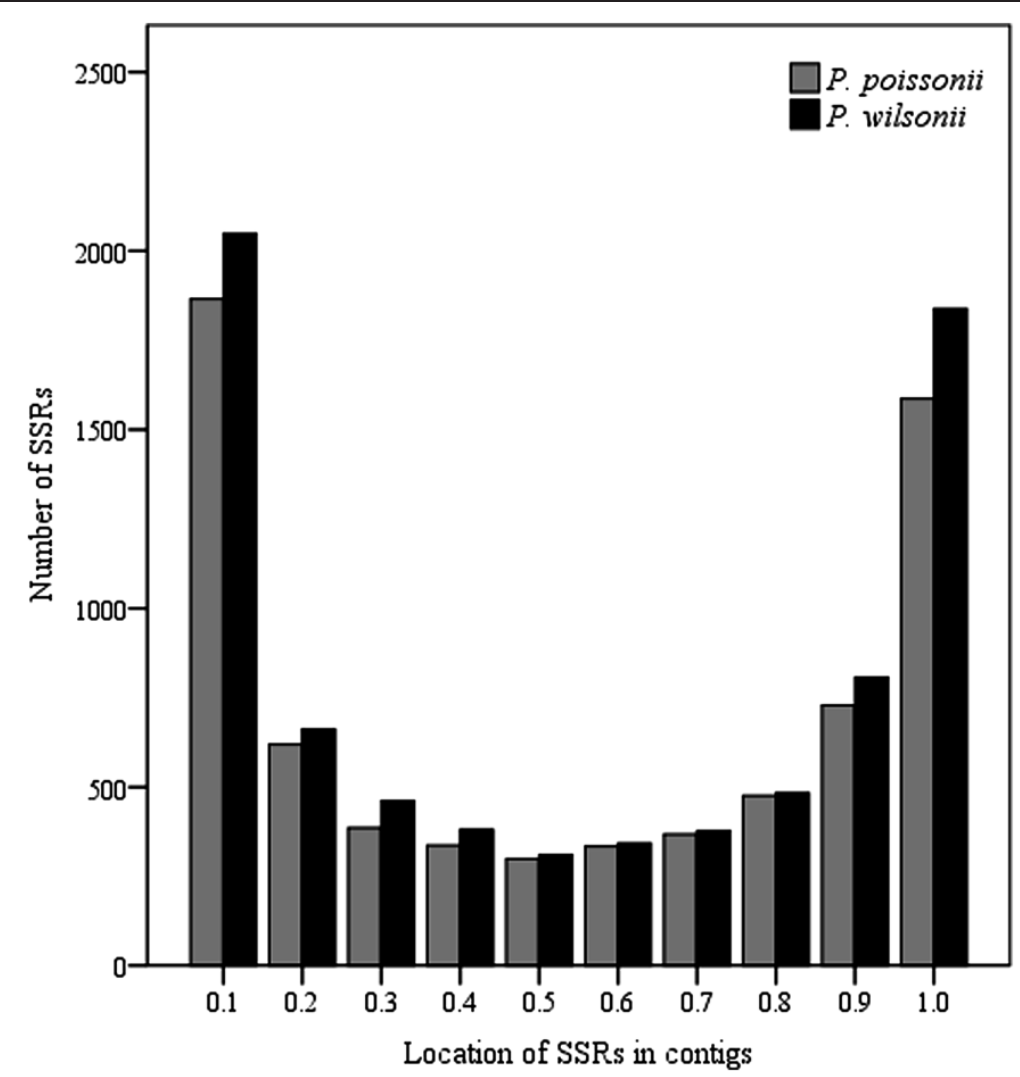

Figure 6 The location distribution of SSRs for the two Primula species; the location is defined by the ratio of the start site position to the total length of the SSR-containing contigs. Grey bar, P. poissonii; black bar, P. wilsonii.

has its own advantages, were applied for characterizing population structure, parentage analysis, genotyping, gene flow inferences and phylogenetic construction. The large number of novel single nuclear gene will greatly increase the resolution of phylogenetic reconstruction for this adaptive taxon. Moreover, these markers with their diverse evolutionary rates will provide unprecedented opportunities to answer the following important questions: What demographic histories underlie the phylogeographic patterns of Primula species? Which evolutionary forces drive the explosive radiation of Primula species in the extreme habitats?

\section{Conclusions}

In this study, we characterized the transcriptomes for the two Primula species, and obtained an unprecedented amount of genomic resources for these important garden plants. Evolutionary analysis of these two species not only yielded a more precise divergence time, but also provided some novel insights into how differential adaptations occurred in extreme habitats. In addition, we developed two sets of genetic markers of popular types, single copy nuclear genes and nuclear microsatellites (EST-SSR). These marker sets will facilitate studies on the genetic improvement, population genetics and phylogenetics of this rapidly adapting taxon.

\section{Methods}

Plant material, RNA extraction and sequencing

Primula poissonii $(2 \mathrm{n}=22)$ is widespread in the mountain areas of northwest Yunnan and southwest Sichuan, China. It inhabits alpine meadows at an altitude of 3200-3500 m. Primula wilsonii $(2 \mathrm{n}=22)$ is distributed in central Yunnan and grows on open lands in evergreen broad-leaved forest at an altitude of ca. $2500 \mathrm{~m}$ [8]. During July 2011, we sampled $P$. poissonii from Zhongdian plateau in northwest Yunnan $\left(28^{\circ} 06^{\prime} 55.24^{\prime \prime} \mathrm{N}, 99^{\circ} 47^{\prime} 48.49^{\prime \prime} \mathrm{E}\right.$, alt. $3314 \mathrm{~m}$ ), and $P$. wilsonii from Ailao Mountain in cen-

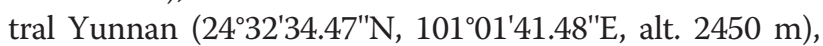
respectively, and fresh leaves and whole flowers of multiple individual plants for both species were stored in RNAlater solution (Takara Biotechnology Co. Ltd., Dalian, China) to preserve the RNA state for use immediately after harvesting. After mixing an approximately equivalent weight of fresh leaves and flowers, total RNA was extracted using a modified CTAB method and precipitated with $5 \mathrm{M}$ $\mathrm{LiCl}_{2}$ at $-20^{\circ} \mathrm{C}$ overnight, and the resulting RNA pellets were suspended in about $100 \mu \mathrm{LEPC}$-treated water. After 

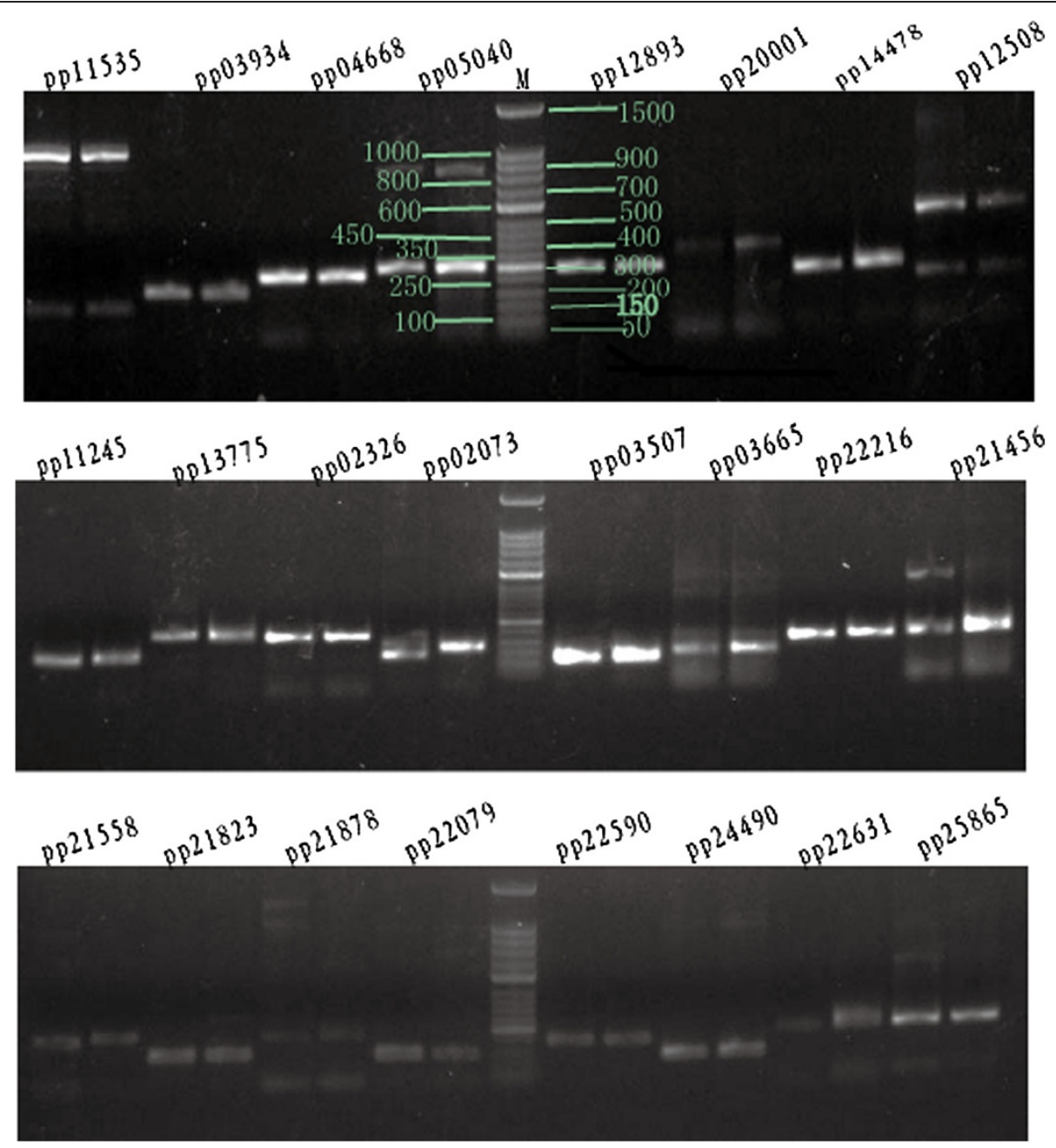

Figure 7 Validation of a subset of the microsatellite primer pairs for the two Primula species by agarose-gel profiling. Results are shown for one individual from each species with pairs name on top; the band profiles for the $50 \mathrm{bp}$ maker ladder are illustrated; additional bands over $300 \mathrm{bp}$ for some primer pairs are non-specific products.

assessing RNA quality by means of electrophoresis and an Eppendorf AG 2231 BioPhotometer Plus (Hamburg, Germany), quantified total RNA (concentration $\geq 100 \mathrm{ng} / \mu \mathrm{L}$; rRNA ratio $\geq 1.5$ ) were delivered to The Beijing Genome Institute (Shenzhen, China) for further treatments. The cDNA library for transcriptome sequencing was prepared using a cDNA Synthesis Kit (Illumina Inc., San Diego, CA, USA) following the manufacturer's recommendations. The cDNA library was then sequenced using a HiSeq2000 (Illumina Inc, San Diego, CA, USA) to obtain short sequences of $90 \mathrm{bp}$ from both ends of each cDNA.

\section{Sequence cleaning, assembly, contig annotation}

Raw reads were firstly subjected to cleaning by removal of adaptors, reads with too many Ns, and reads with quality scores lower than 20. The cleaned reads were assembled de novo using Trinity [36] with the default parameters and contigs with length less than $200 \mathrm{bp}$ were discarded due to a low annotation rate [38]. The filtered reads for $P$. poissonii and $P$. wilsonii were deposited in the NCBI Sequence Read Archive (SRA) under the accession number SRR629689 and SRR640158, respectively.

Functional annotation was implemented using the online program Blast2GO v.2.6.0 [39]. All the assembled contigs were firstly subjected to BLASTX against the NCBI's non-redundant protein database with an E-value threshold of 1E-6. The predicted gene name for each contig was assigned according to the best BLASTX hit. Gene Ontology [40] terms were retrieved from BLASTX hits at E-value threshold 1E-6. Finally, the distributions of level-2 GO terms for all contigs were plotted with the program WEGO [41]. In addition, we download the Vitis 
vinfera proteome [15], and queried against all the assembled contigs using TBLASTN with an E-value threshold of $1 \mathrm{E}-10$ [42].

\section{Identification of orthologous contigs and estimation of substitution rates}

The reciprocal best matches (RBM) method [43] is widely used for identifying orthologs, and a modified version program RBM triangulation [16], allows a third species to be incorporated, which can increase reliability and detect large numbers of conserved orthologs, so we used the following approach for this study. First, we used BLASTN with the RBM method to find orthologs between the two Primula species setting the E-value cutoff at $1 \mathrm{E}-10$. Next, to avoid misspecification caused by the absence of a true ortholog from either Primula species, the third species Vitis was added as positive control. All the reciprocal best hit orthologs were subjected to BLASTX against the Vistis peptide sequences at a threshold E-value of 1E-10, and only those pairs of orthologs with the same reciprocal best hit with Vitis were kept.

With the Vitis peptide sequence as reference, 5'UTR or 3'UTR sequences for some Primula contigs were determined. According to the best-match Vitis peptide sequences, the coding region sequences (CDS) of all the contigs were extracted with custom Perl scripts, and subsequently aligned using the MUSCLE algorithm [44] implemented in MEGA5 [45]. Alignments with unexpected stop codons, or less than 150 base pairs in length, were discarded after checking manually. For the remaining orthologs, synonymous substitution rates (Ks) and non-synonymous rates $(\mathrm{Ka})$ were estimated using a maximum-likelihood method [46] implemented by yn00 in the PAML toolkit [47]. For the closely related species pair, P. poissonii and P. wilsonii, orthologs with $\mathrm{Ks}>0.1$ were excluded to avoid paralogs [48]. Divergence in the CDS sites and UTRs were calculated using the K2P model with a custom Perl script.

On the basis of the Ka/Ks value, setting a threshold at 0.5 , the orthologs were sub-categorized into two dataset: a test set with $\mathrm{Ka} / \mathrm{Ks}$ above 0.5 , and a reference dataset with $\mathrm{Ka} / \mathrm{Ks}$ value less than 0.5 . The significance of the difference in GO term abundance between the two datasets was tested using the Fisher's exact test with the GOSSIP package [49] implemented in BLAST2GO V.2.6.0 [39].

\section{Simple sequence repeats (SSRs) identification and mining} of single copy nuclear genes

The program MISA (http://pgrc.ipk-gatersleben.de/misa/) [50] was used to identify and localize microsatellite motifs in the two Primula species, and only those contigs with motifs containing at least five repeats were selected. The alignments of 6,631 pairs of orthologs were extracted as the input file for the MISA program. Using the detailed information on SSR loci obtained from the output of the MISA program, primers for each SSR-containing sequence with a repetitive at least $16 \mathrm{bp}$ in length were designed with Program Primer Premier 5 (PREMIER Biosoft Int., Palo Alto, CA). To validate the SSRs identified in silico identified SSRs, primer pairs shared between the two Primula species were synthesized (Invitrogen Trading Shanghai Co., Ltd, Shanghai, China), and amplified with one individual of each species as templates. PCRs were performed in a $25 \mu \mathrm{l}$ volume containing $25 \mathrm{ng}$ of template genomic DNA. The PCR reactions were carried out under the following conditions: initial denaturation at $95^{\circ} \mathrm{C}$ for $2 \mathrm{~min}, 35$ cycles at an annealing temperature ranging from $45 \sim 60^{\circ} \mathrm{C}$ for $50 \mathrm{~s}$, and a final extension at $72^{\circ} \mathrm{C}$ for $10 \mathrm{~min}$. The PCR products were checked on $1.5 \%$ agarose gel.

Duarte et al. (2010) identified about 959 sets of single copy nuclear genes shared by Arabidopsis, Populus, Vitis and Oryza (known as APVO genes). We extracted the protein sequences encoded by the APVO gene from the TAIR10 database and queried them against the Primula orthologous EST database using TBLASTN [42] with a threshold E-value of 1E-10. All the queries with hits were considered to be single copy nuclear genes in the Primula species, and the consensus contigs of bestmatched orthologous pairs of the two Primula species were extracted for degenerate primer design using the SeqMan 5.0 program (DNASTAR Inc, Madison, WI, USA). The consensus sequences were queried against the Arabidopsis thaliana protein database using BLASTX with an identity threshold of above 0.75 , then subjected to exon-anchoring and intron-spanning primer design according to the corresponding Arabidopsis thaliana gene models with Program Primer Premier 5 (PREMIER Biosoft Int., Palo Alto, CA, USA). To validate these primers, four of them were randomly chosen to amplify with genomic samples from one individual of each of the two Primula species, and the products were sequenced.

\section{Additional file}

Additional file 1: Table S1. Candidate orthologs under positive selection between $P$. wilsonii and P. poissonii. Table S2. Characteristics of conserved microsatellites primers based on two Primula orthologs. Table S3. Characteristics of primers for Primula-specific single copy nuclear genes homologous to APVO gene sets.

\section{Competing interests}

The authors declared that they have no competing interest.

Authors' contributions

XJG and WW conceived and designed the project. WW and HFY performed the experiments. LZ, WW, XJG analysed and interpreted the data. LZ, WW and XJG drafted the manuscript. HFY and HY revised the manuscript. All authors read and approved the final manuscript. 


\section{Acknowledgements}

We thanks Dr Nolan Kane (University of British Columbia, Canada) for providing data on substitution rate estimations for Asteraceae, thanks are also given to the members of the Adaptive Evolution Lab in Sun Yet-sen University (Guangzhou, China) for helps with bioinformatics analysis. We'd really like to thank Dr. Joelle Hoggan for helping us polish the manuscript This work is financially supported by the National Natural Science Foundation of China (Grant Nos. 31170205, 31270009).

\section{Author details}

'Key Laboratory of Plant Resource Conservation and Sustainable Utilization, South China Botanical Garden, Chinese Academy of Science, Guangzhou 510650, China. ${ }^{2}$ University of Chinese Academy of Sciences, Beijing, China.

Received: 13 December 2012 Accepted: 11 May 2013 Published: 14 May 2013

\section{References}

1. Givnish T: Adaptive radiation and molecular systematic: issues and approaches. In Molecular Evolution and Adaptive Radiation. Edited by Givnish T, Sytsma K. Cambridge, UK: Cambridge University Press; 1997.

2. Elmer KR, Fan S, Gunter HM, Jones JC, Boekhoff S, Kuraku S, Meyer A: Rapid evolution and selection inferred from the transcriptomes of sympatric crater lake cichlid fishes. Mol Ecol 2010, 19(Suppl 1):197-211.

3. Hudson ME: Sequencing breakthroughs for genomic ecology and evolutionary biology. Mol Ecol Resour 2008, 8:3-17.

4. Strickler SR, Bombarely A, Mueller LA: Designing a transcriptome nextgeneration sequencing project for a nonmodel plant species. Am J Bot 2012, 99:257-266

5. Narina SS, Buyyarapu R, Kottapalli KR, Sartie AM, Ali MI, Robert A, Hodeba MJ, Sayre BL, Scheffler BE: Generation and analysis of expressed sequence tags (ESTs) for marker development in yam (Dioscorea alata L.). BMC Genomics 2011, 12:100.

6. Logacheva MD, Kasianov AS, Vinogradov DV, Samigullin TH, Gelfand MS, Makeev VJ, Penin AA: De novo sequencing and characterization of floral transcriptome in two species of buckwheat (Fagopyrum). BMC Genomics 2011, 12:30.

7. Bajgain P, Richardson BA, Price JC, Cronn RC, Udall JA: Transcriptome characterization and polymorphism detection between subspecies of big sagebrush (Artemisia tridentata). BMC Genomics 2011, 12:370.

8. Richards AJ: Primula. 2nd edition. London: B. T. Batsford; 2002

9. Hu CM, Kelso S: Primulaceae. In Flora of China, Volume 15. Edited by Wu ZY, Raven PH. Beijing: St. Louis: Science Press, Missouri Botanical Garden Press; 1996:39-189.

10. Xue DW, Ge XJ, Hao G, Zhang CQ: High genetic diversity in a rare, narrowly endemic primrose species: Primula interjacens by ISSR analysis. Acta Bot $\operatorname{Sin}$ 2004, 46:1163-1169.

11. Van Geert A, Van Rossum F, Stiers I, Sierens T, Barker JHA, Triest L: Isolation and characterization of microsatellite loci in primrose (Primula vulgaris). Belg J Bot 2006, 139:261-264

12. Yan $\mathrm{HF}, \mathrm{Ge} X \mathrm{XJ}, \mathrm{Hu} \mathrm{CM}, \mathrm{Hao} \mathrm{G}$ : Isolation and characterization of microsatellite loci for the ornamental plant Primula obconica Hance (Primulaceae). Hortscience 2010, 45:314-315.

13. Ueno S, Yoshida Y, Taguchi Y, Ujino-Ihara T, Kitamoto N, Honjo M, Ohsawa R, Washitani I, Tsumura Y: Generation of expressed sequence tags, development of microsatellite and single nucleotide polymorphism markers in Primula sieboldii E. Morren (Primulaceae) for analysis of genetic diversity in natural and horticultural populations. Breeding Sci 2011, 61:234-243.

14. Hu CM: Primulaceae. In Flora Reipublicae Popularis Sinicae, Volume 59(2). Beijing: Science Press; 1990:113-128.

15. Jaillon O, Aury JM, Noel B, Policriti A, Clepet C, Casagrande A, Choisne N Aubourg S, Vitulo N, Jubin $C$, et al: The grapevine genome sequence suggests ancestral hexaploidization in major angiosperm phyla. Nature 2007, 449:463-467.

16. Wu F, Mueller LA, Crouzillat D, Petiard V, Tanksley SD: Combining bioinformatics and phylogenetics to identify large sets of single-copy orthologous genes (COSII) for comparative, evolutionary and systematic studies: a test case in the euasterid plant clade. Genetics 2006 174:1407-1420.
17. Makalowski W, Boguski MS: Evolutionary parameters of the transcribed mammalian genome: an analysis of 2,820 orthologous rodent and human sequences. Proc Natl Acad Sci USA 1998, 95:9407-9412

18. Swanson WJ, Wong A, Wolfner MF, Aquadro CF: Evolutionary expressed sequence tag analysis of Drosophila female reproductive tracts identifies genes subjected to positive selection. Genetics 2004, 168:1457-1465.

19. Wang $Y$, Hey J: Estimating divergence parameters with small samples from a large number of loci. Genetics 2010, 184:363-379.

20. Blanc $\mathrm{G}$, Wolfe $\mathrm{KH}$ : Widespread paleopolyploidy in model plant species inferred from age distributions of duplicate genes. Plant Cell 2004, 16:1667-1678.

21. Kane NC, Barker MS, Zhan SH, Rieseberg LH: Molecular evolution across the Asteraceae: micro- and macroevolutionary processes. Mol Biol Evol 2011, 28:3225-3235

22. Graur D, Li WH: Fundamentals of Molecular Evolution. 2nd edition. Tel Aviv, Israel: Sinauer Associates; 2000.

23. Koch MA, Haubold B, Mitchell-Olds T: Comparative evolutionary analysis of chalcone synthase and alcohol dehydrogenase loci in Arabidopsis, Arabis, and related genera (Brassicaceae). Mol Biol Evol 2000, 17:1483-1498.

24. Lynch M, Conery JS: The evolutionary fate and consequences of duplicate genes. Science 2000, 290:1151-1155.

25. Tamura K, Adachi Y, Chiba K, Oguchi K, Takahashi H: Identification of Ku70 and Ku80 homologues in Arabidopsis thaliana: evidence for a role in the repair of DNA double-strand breaks. Plant J 2002, 29:771-781.

26. Xia R, Wang J, Liu C, Wang Y, Zhai J, Liu J, Hong X, Cao X, Zhu JK, Gong Z: ROR1/RPA2A, a putative replication protein $\mathrm{A} 2$, functions in epigenetic gene silencing and in regulation of meristem development in Arabidopsis. Plant Cell 2006, 18:85-103.

27. Watanabe K, Pacher M, Dukowic S, Schubert V, Puchta H, Schubert I: The STRUCTURAL MAINTENANCE OF CHROMOSOMES $5 / 6$ complex promotes sister chromatid alignment and homologous recombination after DNA damage in Arabidopsis thaliana. Plant Cell 2009, 21:2688-2699.

28. Takahashi N, Quimbaya M, Schubert V, Lammens T, Vandepoele K, Schubert I, Matsui M, Inze D, BerX G, De Veylder L: The MCM-binding protein ETG1 aids sister chromatid cohesion required for postreplicative homologous recombination repair. PLoS Genet 2010, 6:e1000817.

29. Chang CC, Slesak I, Jorda L, Sotnikov A, Melzer M, Miszalski Z, Mullineaux PM, Parker JE, Karpinska B, Karpinski S: Arabidopsis chloroplastic glutathione peroxidases play a role in cross talk between photooxidative stress and immune responses. Plant Physiol 2009, 150:670-683.

30. Li $L$, Ren F, Gao XQ, Wei PC, Wang XC: The reorganization of actin filaments is required for vacuolar fusion of guard cells during stomatal opening in Arabidopsis. Plant Cell Environ 2013, 36:484-497.

31. Zhang H, Ohyama K, Boudet J, Chen Z, Yang J, Zhang M, Muranaka T, Maurel C, Zhu JK, Gong Z: Dolichol biosynthesis and its effects on the unfolded protein response and abiotic stress resistance in Arabidopsis. Plant Cell 2008, 20:1879-1898

32. Dutilleul C, Jourdain A, Bourguignon J, Hugouvieux V: The Arabidopsis putative selenium-binding protein family: expression study and characterization of SBP1 as a potential new player in cadmium detoxification processes. Plant Physiol 2008, 147:239-251.

33. Ravet K, Touraine B, Kim SA, Cellier F, Thomine S, Guerinot ML, Briat JF, Gaymard F: Post-translational regulation of AtFER2 ferritin in response to intracellular iron trafficking during fruit development in Arabidopsis. Mol Plant 2009, 2:1095-1106.

34. Bowman JL, Smyth DR, Meyerowitz EM: Genes directing flower development in Arabidopsis. Plant Cell 1989, 1:37-52.

35. Mehrtens F, Kranz H, Bednarek P, Weisshaar B: The Arabidopsis transcription factor MYB12 is a flavonol-specific regulator of phenylpropanoid biosynthesis. Plant Physiol 2005, 138:1083-1096.

36. Grabherr MG, Haas BJ, Yassour M, Levin JZ, Thompson DA, Amit I, Adiconis X, Fan $L$, Raychowdhury R, Zeng $Q$, et al: Full-length transcriptome assembly from RNA-Seq data without a reference genome. Nat Biotechnol 2011, 29:644-652.

37. Duarte JM, Wall PK, Edger PP, Landherr LL, Ma H, Pires JC, Leebens-Mack J, de Pamphilis CW: Identification of shared single copy nuclear genes in Arabidopsis, Populus, Vitis and Oryza and their phylogenetic utility across various taxonomic levels. BMC Evol Biol 2010, 10:61.

38. Gotz S, Garcia-Gomez JM, Terol J, Williams TD, Nagaraj SH, Nueda MJ, Robles M, Talon M, Dopazo J, Conesa A: High-throughput functional annotation and data mining with the Blast2GO suite. Nucleic Acids Res 2008, 36:3420-3435. 
39. Conesa A, Gotz S, Garcia-Gomez JM, Terol J, Talon M, Robles M: Blast2GO: a universal tool for annotation, visualization and analysis in functional genomics research. Bioinformatics 2005, 21:3674-3676.

40. Ashburner M, Ball CA, Blake JA, Botstein D, Butler H, Cherry JM, Davis AP, Dolinski K, Dwight SS, Eppig JT, et al: Gene ontology: tool for the unification of biology. Nat Genet 2000, 25:25-29.

41. Ye J, Fang L, Zheng H, Zhang Y, Chen J, Zhang Z, Wang J, Li S, Li R, Bolund L: WEGO: a web tool for plotting GO annotations. Nucleic Acids Res 2006, 34(Web Server issue):W293-W297.

42. Altschul SF, Madden TL, Schaffer AA, Zhang JH, Zhang Z, Miller W, Lipman DJ: Gapped BLAST and PSI-BLAST: a new generation of protein database search programs. Nucleic Acids Res 1997, 25:3389-3402.

43. Li L, Stoeckert CJ Jr, Roos DS: OrthoMCL: identification of ortholog groups for eukaryotic genomes. Genome Res 2003, 13:2178-2189.

44. Edgar RC: MUSCLE: multiple sequence alignment with high accuracy and high throughput. Nucleic Acids Res 2004, 32:1792-1797.

45. Tamura K, Peterson D, Peterson N, Stecher G, Nei M, Kumar S: MEGA5: molecular evolutionary genetics analysis using maximum likelihood, evolutionary distance, and maximum parsimony methods. Mol Biol Evol 2011, 28:2731-2739

46. Yang $\mathrm{ZH}$, Nielsen R: Estimating synonymous and nonsynonymous substitution rates under realistic evolutionary models. Mol Biol Evol 2000, 17:32-43.

47. Yang Z: PAML 4: phylogenetic analysis by maximum likelihood. Mol Biol Evol 2007, 24:1586-1591.

48. Bustamante CD, Fledel-Alon A, Williamson S, Nielsen R, Hubisz MT, Glanowski S, Tanenbaum DM, White TJ, Sninsky JJ, Hernandez RD, et al: Natural selection on protein-coding genes in the human genome. Nature 2005, 437:1153-1157.

49. Bluthgen N, Brand K, Cajavec B, Swat M, Herzel H, Beule D: Biological profiling of gene groups utilizing Gene Ontology. Genome Inform 2005, 16:106-115.

50. Sharma PC, Grover A, Kahl G: Mining microsatellites in eukaryotic genomes. Trends Biotechnol 2007, 25:490-498.

doi:10.1186/1471-2164-14-329

Cite this article as: Zhang et al:: Comparative transcriptome analysis and marker development of two closely related Primrose species (Primula poissonii and Primula wilsonii). BMC Genomics 2013 14:329.

\section{Submit your next manuscript to BioMed Central and take full advantage of:}

- Convenient online submission

- Thorough peer review

- No space constraints or color figure charges

- Immediate publication on acceptance

- Inclusion in PubMed, CAS, Scopus and Google Scholar

- Research which is freely available for redistribution 